\title{
Clinical Treatment Efficacy Using One-snip Punctoplasty and Irrigation Technique in Primary Canaliculitis Patients
}

\author{
Ui Seo Park, Jae Wook Yang, Young Jin Kim \\ Department of Ophthalmology, Inje University Busan Paik Hospital, Inje University College of Medicine, Busan, Korea
}

\begin{abstract}
Purpose: This study aims to report the efficacy and safety of one-snip punctoplasty and 18-gauge irrigation technique in patients with primary canaliculitis.

Methods: All patients diagnosed with primary canaliculitis between January 2020 and August 2021 at Inje University Busan Paik Hospital are included. All patients underwent one-snip punctoplasty and 18-gauge irrigation technique. After the procedure, patients had topical antibiotics. The resolution of symptoms and inflammatory signs and complications were evaluated 3 weeks after the procedure.
\end{abstract}

Results: A total of 11 patients (eight female patients and three male patients, 14 canaliculi) aged 34 to 82 years with a mean age of $63.8 \pm 15.7$ years were participated. Common symptoms were epiphora, mucopurulent discharge, and injection, and common signs were discharge from punctum, pouting punctum, punctal erythema, and swelling. Among 14 canaliculi, 12 (85.7\%) had complete resolution and two underwent second treatment which showed completed resolution after the treatment.

Conclusions: One-snip punctoplasty and 18-gauge irrigation technique are minimally invasive to punctum and canaliculi and are a highly effective surgical procedure for patients with primary canaliculitis.

Key Words: Irrigation technique, One-snip punctoplasty, Primary canaliculitis

Primary canaliculitis is characterized as an infection of the proximal lacrimal pathway that accounts for approximately $1.4 \%$ to $2 \%$ of all lacrimal diseases [1]. Patients with canaliculitis typically present symptoms including mucopurulent discharge, epiphora, and pouting punctum. It is easily misdiagnosed as chronic conjunctivitis, chalazion or

Received: January 21, 2022

Accepted: February 4, 2022

Corresponding Author: Young Jin Kim, MD. Department of Ophthalmology, Inje University Busan Paik Hospital, 75 Bokjin-ro, Busanjin-gu, Busan 47392, Korea. Tel: 82-051-890-6015, Fax: 82-051-890-6329, E-mail: italia08@naver.com dacryocystitis [2], which may lead to delayed or insufficient treatment [3]. Medical treatment alone with topical or systemic antibiotics is not sufficient and sulphur granules and granulation tissue must be removed. Various surgical techniques to accomplish this have been reported.

Canaliculotomy is a widely used surgical technique for primary canaliculitis. However, canaliculotomy is an invasive procedure which may result in canalicular obstruction or lacrimal pump dysfunction and epiphora in $20 \%$ to $25 \%$ of the patients [3-5]. Therefore, many surgical methods have been reported to reduce the complications.

Lee et al. [6] reported that one-snip punctoplasy and canalicular curettage through the punctum had a success 
rate of $83.3 \%$. Lee et al. [7] reported squeezing the punctum and lacrimal canaliculus using cotton tips, while Kim et al. [8] reported using a four-snip punctoplasty and canalicular curettage for recurrent primary canaliculitis. In this study, we will be reporting our results with the one-snip punctoplasty and irrigation technique, which includes a punctal dilatation and one-snip punctoplasty procedure, compression of the canaliculus to express the sulphur granules, and irrigation with an 18-gauge irrigation tip while compressing the medial canthus.

\section{Materials and Methods}

This study is approved by the institutional review board of Inje University Busan Paik Hospital (No. BPIRB 202110-028-003). The need for written consent was waived due to the retrospective nature of this study.

The medical records of all patients treated for primary canaliculitis between January 2020 and August 2021 at Inje University Busan Paik Hospital were reviewed. The patient demographics, symptoms, and signs were investigated.
Postoperative symptoms and procedure outcomes were collected as well as sign and slit lamp findings. Diagnostic criteria were the presence of swelling and erythema of the affected punctum and canaliculus, a pouting punctum, and mucopurulent punctal discharge. Patients with secondary canaliculitis, such as punctal plug induced canaliculitis or silicon tube induced canaliculitis, previous lacrimal surgery, and combined ocular disease, were excluded. All patients were treated by a single oculoplastics surgeon.

Following local anesthesia with $2 \%$ lidocaine mixed with epinephrine, one-snip punctoplaty was performed. A single vertical cut was made along the posterior wall of the punctum on the conjunctival side with mini Wescott scissors. (Fig. 1A) Punctum and lacrimal canaliculi were squeezed using cotton tips to remove the sulphur granule until no canalicular contents came out. (Fig. 1B) While compressing medial canthus, the infected canaliculi were irrigated using an 18-gauge irrigation tip (Fig. 1C) until no more sulphur granules appeared (Fig. 2A-2C). After surgery, all patients were prescribed a moxifloxacin drop four times a day for three weeks.

Three weeks after the surgery, all patients underwent
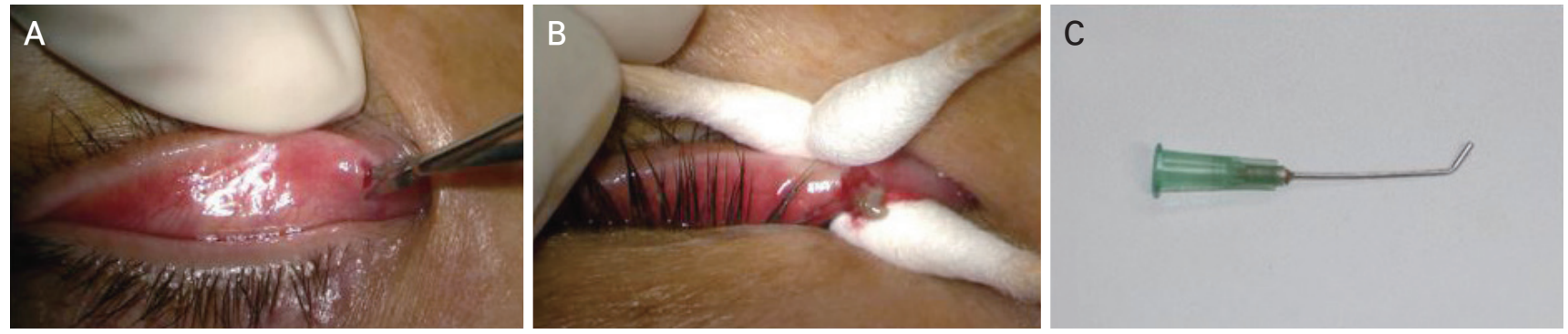

Fig. 1. Procedure of one-snip punctoplasty and a picture of 18-gauge needle. (A) Single vertical cut was made along the posterior wall of punctum on conjunvtival side with a mini Wescott scissor. (B) Punctum and lacrimal canaliculi were squeezed using cotton tip to remove sulphur granule. (C) An 18-gauge irrigation tip used to perform irrigation technique.
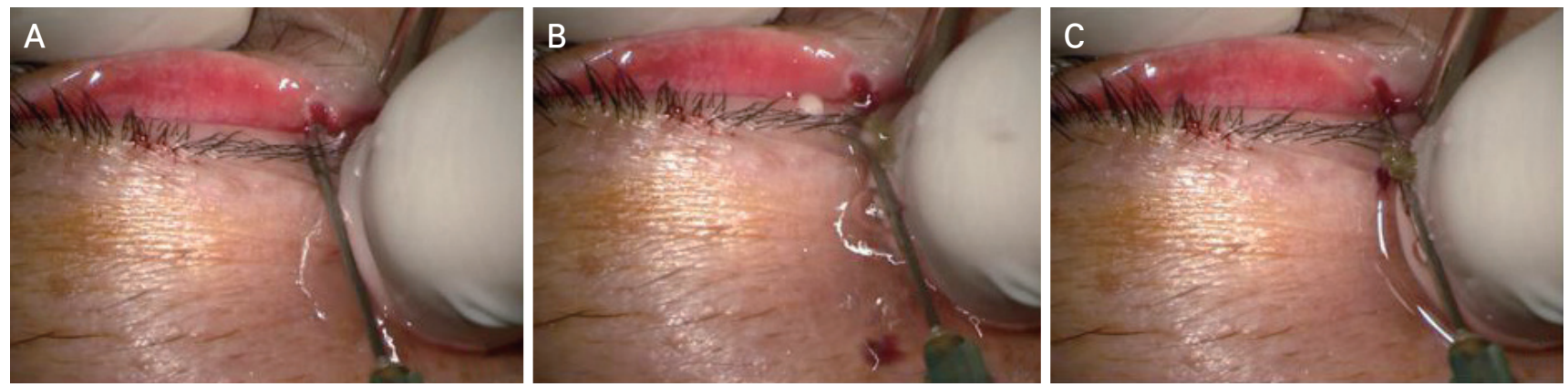

Fig. 2. Procedure of irrigation technique. $(A, B)$ While compressing medial canthus, infected canaliculi were irrigated using 18 -gauge irrigation tip. (C) Irrigation was performed until no more sulphur granule appeared. 
follow-up examinations with the specific purpose to confirm whether the presenting symptoms and signs were resolved and if new symptoms occurred. Punctum were also investigated.

\section{Results}

Eleven patients with 14 infected canaliculi underwent a one-snip punctoplasty and irrigation technique procedure. Consisting of eight female patients and three male patients aged 34 to 82 years with a mean age of $63.8 \pm 15.7$ years, seven patients had only left eye involvement, three had

Table 1. Clinical characteristics of patients

\begin{tabular}{lc}
\hline Characteristics & Value \\
\hline Sex (male : female) & $3: 8(27.3: 72.7)$ \\
Age (yr) & $63.8 \pm 15.7(34-82)$ \\
Mean time to diagnosis (mon) & $12.3 \pm 18.6(1 \mathrm{wk}-5 \mathrm{yr})$ \\
Location (upper : lower : both) & $1: 8: 2(1: 72.7: 18)$ \\
Laterality (right : left : both) & $3: 7: 1(27.3: 64.6: 9.1)$ \\
Mean follow-up (mon) & $3.27 \pm 2.35(3 \mathrm{wk}-7$ mon $)$ \\
\hline
\end{tabular}

Values are presented as number (\%) or mean \pm standard deviation (range).

Table 2. Clinical presentations of patients before treatment

\begin{tabular}{lc}
\hline Clinical presentations & No. $(\%)$ \\
\hline Symptoms & \\
Epiphora & $4(36.4)$ \\
Mucopurulent discharge & $11(100.0)$ \\
Conjunctival injection & $5(45.5)$ \\
Signs & \\
Discharge from punctum & $11(100.0)$ \\
Pouting punctum & $8(72.7)$ \\
Punctal erythema and swelling & $3(27.3)$ \\
\hline
\end{tabular}

only right eye involvement, and one had both eyes. One patient had upper canaliculitis and eight patients had lower canaliculitis, while two patients had both upper and lower canaliculitis. Their clinical characteristics are summarized in Table 1. The mean time from the first onset of symptoms/signs to diagnosis was $12.3 \pm 18.6$ weeks ranging from 1 week to 5 years. The presenting symptoms and clinical signs are summarized in Table 2. The most common presentations were mucopurulent discharge (11 patients, $100 \%$ ). All patients had expressible discharge from the punctum. There were no patients who had mucous reflux on the lacrimal sac when it was compressed.

The follow-up time after surgery ranged from 3 weeks to 7 months with a mean time of $3.27 \pm 2.35$ months. Three weeks after initial surgery, complete remission of their clinical symptoms and signs were noted in 12 of the 14 canaliculi (85.7\%) (Table 3). In the remaining two canaliculi, there was remaining mucopurulent discharge, erythema, and swelling. All canaliculi were treated with second one-snip punctoplasty with an irrigation technique procedure. The remaining sulphur granules were evacuated, all of which were in the lateral side of punctum and not in the medial side of the infected canaliculi. There were no signs or symptoms of canaliculitis 3 weeks after the second procedure. During follow-up visits, no patients developed a recurrence of canaliculitis after their second procedure.

Table 3. Success rate and second treatment after the treatment

\begin{tabular}{lc}
\hline Success rate & No. $(\%)$ \\
\hline First treatment & \\
Complete resolution & $12(85.7)$ \\
Persistent symptom & $2(14.3)$ \\
Second treatment & \\
Complete resolution & $2(100.0)$ \\
Persistent symptom & $0(0)$ \\
\hline
\end{tabular}

Two had secondary treatment and had complete resolution after the treatment.

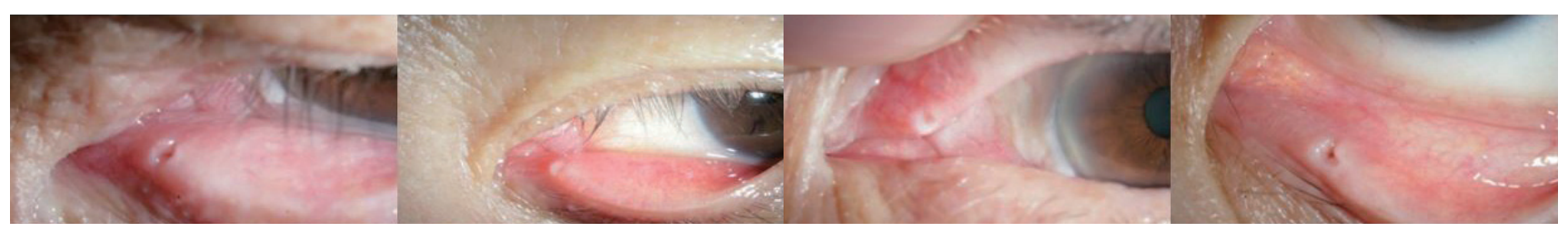

Fig. 3. Picture of puncta 3 weeks after the procedure. All pucnta showed no positional or structural changes such as fibrosis or scarring 
Three weeks after first and second procedure, all patients had no additional lacrimal symptom such as epiphora. There were no positional or structural change of punctum such as stricture, fibrosis, or scarring (Fig. 3).

\section{Discussion}

Lee et al. [7] reported that only squeezing punctum and lacrimal canaliculi using cotton tips is an effective treatment for the canaliculitis with a success rate of $71.4 \%$ and without causing complications in the canalicular system. There were no complications with the lacrimal system associated with punctum and canaliculus because they did not perform curettage and manipulation of punctum such as punctoplasty and canaliculotomy. However, the success rate $(71.4 \%)$ was relatively lower than other studies. Only squeezing punctum and lacrimal canaliculi can have limitations for removing all of the canalicular contents. Therefore, curettage was commonly performed. Lee et al. [6] performed one-snip punctoplasty and canalicular curettage with a success rate of $83.3 \%$. They reported two patients had newly developed epiphora with canalicular stricture after one-snip punctoplasty and curettage. The incidence of changes in canaliculi structures remains unknown after surgical procedures such as canaliculotomy, canalicular curettage, punctoplasty, and canaliculoplasty.

Canaliculotomy, an invasive procedure, can cause canalicular obstruction. Vescei et al. [3] reported $26.6 \%$ of patients had epiphora after canaliculotomy. Anand et al. [5] reported epiphora in four of 15 patients after canaliculotomy in their study. Canaliculotomy may potentially cause canalicular obstruction or stricture due to fibrosis, scarring, and fistula formation. Due to canaliculus having dynamic mechanical properties, canaliculotomy can cause lacrimal pump dysfunction and epiphora even in patients with intact lacrimal outflow with irrigation examination. $\mathrm{Su}$ et al. [9] introduced canaliculoplasty to reconstruct an anatomical structure of canaliculus, but nine patients (of 43) had epiphora and three of them had complete canalicular obstruction due to scar formation in dacryoendocscopy.

The canalicular curettage can also damage inflamed canalicular mucosa, as well as cause scarring, fibrosis, and granulation. Even if gently performed, metallic curette may potentially cause canalicular obstruction.

In this study, our technique, a one-snip punctoplasty and irrigation technique using an 18-gauge irrigation tip without canalicular curettage, can reduce a damage to lacrimal flow system by minimizing manipulation of the punctum and canaliculi as much as possible. Unlike other studies, which performed curettage, the sulphur granules were evacuated by high hydraulic pressure that was formed by irrigation of normal saline using a thick 18-gauge irrigation tip. After pressing the medial canthus to apply pressure to the common canaliculus and the lacrimal sac, normal saline was irrigated through the canaliculus to remove the sulphur granules remaining in the canaliculus with high hydraulic pressure. As the irrigation tip usually used to exam lacrimal system cannot make sufficient hydraulic pressure, the 18-gauge irrigation tip was used. We also performed a one-snip punctoplasty which can cause sufficient punctal dilatation to remove sulphur granules with minimal damage to punctum. Guerico et al. [10] reported onesnip punctoplasty did not affect the symptoms of epiphora with lower eyelid tightening in patients with lacrimal pump failure. On the other hand, one-snip punctoplasty redirects the punctum slightly posteriorly, which may be beneficial with very mild punctal eversion.

Based on posterior incision of the punctum in two recurrent patients, all of the remaining sulphur granules were in the lateral side of the punctum. To remove them, the irrigation tip was put inside of the medial punctum and irrigated. Following this, the tip was also placed on the lateral side of the punctum and irrigated. Long standing, severe canaliculitis can make the vertical canaliculus and ampulla of punctum dilated, where enough space is created for sulphur granules to exist. The sulphur granules moved from the high hydraulic pressure of normal saline, but the punctum was not dilated enough to pass out, so they remained on the lateral side of the posterior incision of punctum where it caused a recurrence. All of the patients improved their symptoms after the second treatment.

In this study the structure of canaliculus after the procedure could not be thoroughly evaluated because the dacryoendocopy, which allows direct visualization of the lacriaml drainage system, was not routinely performed. However, it can cause injury to canaliculi, especially when the mucosa of canaliculi has not completely healed from inflammation after surgical treatment. In this study, the mean follow-up time was short $(3.27 \pm 2.35$ months, 3 weeks to 7 months). An evaluation of lacrimal drainage system of canaliculitis after procedure was not made. It 
would be preferable to have long-term dacryoendoscopic findings after a procedure in order to evaluate the lacrimal drainage system and promote this procedure unequivocally. This study is also limited by its retrospective nature and the lack of a control group. It is also limited by the small number of patients making it difficult to analyze and compare our procedure with other techniques.

In summary, one-snip punctoplasty and 18-gauge irrigation technique are minimally invasive to punctum and canaliculi and are a highly effective surgical procedure for patients with primary canaliculitis. Careful irrigation of both medial and lateral sides of punctum based on posterior incision to completely remove sulphur granules increases the success rate with minimal canalicular damage.

\section{Conflicts of Interest: None.}

Acknowledgements: None.

Funding: This research was supported by a grant of the Korea Health Technology R\&D Project through the Korea Health Industry Development Institute, funded by the Ministry of Health \& Welfare, Korea (No. HR21C1003).

\section{References}

1. Demant E, Hurwitz JJ. Canaliculitis: review of 12 cases. Can J Ophthalmol 1980;15:73-5.

2. Kaliki S, Ali MJ, Honavar SG, et al. Primary canaliculitis: clinical features, microbiological profile, and management outcome. Ophthalmic Plast Reconstr Surg 2012;28:355-60.

3. Vecsei VP, Huber-Spitzy V, Arocker-Mettinger E, Steinkogler FJ. Canaliculitis: difficulties in diagnosis, differential diagnosis and comparison between conservative and surgical treatment. Ophthalmologica 1994;208:314-7.

4. Zaldivar RA, Bradley EA. Primary canaliculitis. Ophthalmic Plast Reconstr Surg 2009;25:481-4.

5. Anand S, Hollingworth K, Kumar V, Sandramouli S. Canaliculitis: the incidence of long-term epiphora following canaliculotomy. Orbit 2004;23:19-26.

6. Lee MJ, Choung HK, Kim NJ, Khwarg SI. One-snip punctoplasty and canalicular curettage through the punctum: a minimally invasive surgical procedure for primary canaliculitis. Ophthalmology 2009;116:2027-30.

7. Lee HK, Park JH, Lee JS, et al. Therapeutic effect of squeezing the punctum and lacrimal canaliculus in canaliculitis patients. $J$ Korean Ophthalmol Soc 2016;57:700-4.

8. Kim MH, Ra H. A case report on complete cure of recurrent primary canaliculitis by 4 -snip punctoplasty and canalicular curettage. Medicine (Baltimore) 2018;97:e13508.

9. Su Y, Zhang L, Li L, et al. Surgical procedure of canaliculoplasty in the treatment of primary canaliculitis associated with canalicular dilatation. BMC Ophthalmol 2020;20:245.

10. Guercio B, Keyhani K, Weinberg DA. Snip punctoplasty offers little additive benefit to lower eyelid tightening in the treatment of pure lacrimal pump failure. Orbit 2007;26:15-8. 